\title{
MOMENTS AND CENTRAL LIMIT THEOREMS FOR SOME MULTIVARIATE POISSON FUNCTIONALS
}

\author{
GÜNTER LAST, ${ }^{* *}$ Karlsruhe Institute of Technology \\ MATHEW D. PENROSE, ${ }^{* * *}$ University of Bath \\ MATTHIAS SCHULTE, ${ }^{* * * * *}$ Karlsruhe Institute of Technology \\ CHRISTOPH THÄLE, ${ }^{* * * * *}$ Ruhr University Bochum
}

\begin{abstract}
This paper deals with Poisson processes on an arbitrary measurable space. Using a direct approach, we derive formulae for moments and cumulants of a vector of multiple Wiener-Itô integrals with respect to the compensated Poisson process. Also, we present a multivariate central limit theorem for a vector whose components admit a finite chaos expansion of the type of a Poisson $U$-statistic. The approach is based on recent results of Peccati et al. (2010), combining Malliavin calculus and Stein's method; it also yields Berry-Esseen-type bounds. As applications, we discuss moment formulae and central limit theorems for general geometric functionals of intersection processes associated with a stationary Poisson process of $k$-dimensional flats in $\mathbb{R}^{d}$.
\end{abstract}

Keywords: Berry-Esseen-type bound; central limit theorem; intersection process; multiple Wiener-Itô integral; Poisson process; Poisson flat process; product formula; stochastic geometry; Wiener-Itô chaos expansion

2010 Mathematics Subject Classification: Primary 60D05; 60H07

Secondary 60F05; 60G55

\section{Introduction}

Throughout this paper we denote by $(\mathbb{X}, \mathcal{X})$ a measurable space, equipped with a $\sigma$-finite measure $\lambda \neq 0$. The classical Wiener-Itô chaos expansion says that if $F \equiv F(\eta)$ is a square integrable function of a Poisson process $\eta$ on $(\mathbb{X}, \mathcal{X})$ with intensity measure $\lambda$ (see [7, Chapter 12]), then $F$ can be represented as an orthogonal $L^{2}$-series

$$
F=\mathbb{E} F+\sum_{n=1}^{\infty} I_{n}\left(f_{n}\right),
$$

where $I_{n}\left(f_{n}\right)$ is the multiple ( $n$-fold) Wiener-Itô integral of a certain symmetric function $f_{n}: \mathbb{X}^{n} \rightarrow \mathbb{R}$ that is square integrable with respect to $\lambda^{n}$. For a proof of this result without any further assumptions on the measure space $(\mathbb{X}, \mathcal{X}, \lambda)$, we refer the reader to [10]. It turns out that

Received 17 May 2012; revision received 9 July 2013.

* Postal address: Institute of Stochastics, Karlsruhe Institute of Technology, 76128 Karlsruhe, Germany.

** Email address: guenter.last@kit.edu

*** Postal address: Department of Mathematical Sciences, University of Bath, Bath BA2 7AY, UK.

Email address: m.d.penrose@ bath.ac.uk

**** Email address: matthias.schulte@kit.edu

***** Postal address: Faculty of Mathematics, Ruhr University Bochum, 44801 Bochum, Germany.

Email address: christoph.thaele@rub.de 
the chaos expansion (1) is useful for many purposes. For instance, it serves as a mathematical basis for Malliavin calculus of variations on the Poisson space and can be used to formulate and to prove central limit theorems; see [13] and [14].

The present paper deals with multivariate Poisson functionals $\left(F_{t}^{(1)}, \ldots, F_{t}^{(\ell)}\right), \ell \in \mathbb{N}$, where each component is of the form $F_{t}^{(i)}=F^{(i)}\left(\eta_{t}\right)$ and $\eta_{t}$ is a Poisson point process with an intensity measure of the form $\lambda_{t}=t \lambda$. Here, we are interested in the asymptotic regime that arises when the intensity parameter $t$ tends to infinity. Under the additional assumption that each $F_{t}^{(i)}$ is a $U$-statistic of the Poisson process $\eta_{t}$, we prove formulae for the joint moments and cumulants and a multivariate central limit theorem.

The assumption that the functionals $F_{t}^{(i)}$ are Poisson $U$-statistics implies that their chaos expansions (1) terminate after a finite numbers of terms, which is convenient for the application of Malliavin calculus. Univariate central limit theorems with bounds on the Wasserstein distance for Poisson functionals with finite Wiener-Itô chaos expansions and, in particular, Poisson $U$ statistics are derived in [8], [9], and [16] using a general result from [14]. Our multivariate counterpart rests on a multivariate analogue in [13]. By using a new truncation argument and the special form of the Poisson functionals, our approach avoids technical computations involving the product formula for multiple Wiener-Itô integrals that is used in the works mentioned previously.

As an application, we study in detail geometric functionals of the intersection process of order $m \in\{1, \ldots, d\}$ of a stationary Poisson $k$-flat process in $\mathbb{R}^{d}$. We thereby considerably extend the results available in the literature (see [4] and [5]) for the number of intersections and the intersection volume. In our theory we can allow for very general geometric functionals; for example, we do not require them to be additive, translation-invariant, or homogeneous. Furthermore, our central limit theorems are quantitative in the sense that they provide rates of convergence (with respect to a suitable distance).

For the asymptotic analysis of problems in stochastic geometry, two natural limiting regimes (among others) may be considered. On the one hand, we can fix the intensity of the underlying (Poisson) point process and increase the observation window in which everything takes place. On the other hand, we can fix this window and increase the intensity. We emphasize that these two regimes lead to limit theorems of different natures. Only in exceptional cases (such as for homogeneous functionals of Poisson $k$-flat processes considered in Section 6, below), is it possible to derive one limit theorem from the other. Our results deal with the situation of increasing intensity in the case where the functional of interest has the form of a Poisson $U$-statistic. As well as in the context described above, our theory can thus be applied directly to numbers of $k$-simplices of random simplical complexes (see [3]) and to subgraph counting in random geometric graphs (see [8] and [15]) with a fixed distance threshold. For problems that were previously considered in the literature for fixed intensity and increasing observation windows, such as the numbers of $k$-clusters [1], statistics of rather general random geometric graphs [9], or proximity functionals of nonintersecting $k$-flat processes [18], our results provide complementary central limit theorems for fixed windows and increasing intensity.

Another direction this paper deals with is formulae for mixed moments and cumulants, which in turn are based on identities for mixed moments and cumulants of multiple Wiener-Itô integrals. We develop a quick approach to prove these formulae that have previously appeared under different assumptions in [12] and [19]. The novelty of our proof is that it only makes use of elementary properties of the Poisson process (mainly the multivariate Mecke formula) and some combinatorial arguments, and deals directly with the expectation. In this way it avoids requiring the involved chaos expansion of such products. 
This paper is structured as follows. In Section 2 we collect some basic definitions and background material. The moment formulae are presented in Section 3 while Section 4 deals with their asymptotic behaviour. Our multivariate central limit theorems for $U$-statistics of the underlying Poisson process are described in Section 5, while in Section 6 our results are applied to Poisson $k$-flat processes in $\mathbb{R}^{d}$.

\section{Preliminaries}

In this paper all random objects are defined on a probability space $(\Omega, \mathcal{F}, \mathbb{P})$. We interpret the Poisson process $\eta$ as a random element in the space $N:=N(\mathbb{X})$ of integer-valued (including $+\infty) \sigma$-finite measures $\mu$ on $\mathbb{X}$ equipped with the smallest $\sigma$-field $\mathcal{N}$ making the mappings $\mu \mapsto \mu(B)$ measurable for all $B \in \mathcal{X}$. For $m \in \mathbb{N}$ and $\mu \in N$, we define a measure $\mu^{(m)}$ on $\mathbb{X}^{m}$ by

$$
\begin{aligned}
\mu^{(m)}(B):=\int \cdots \int & \mathbf{1}_{B}\left(x_{1}, \ldots, x_{m}\right)\left(\mu-\sum_{j=1}^{m-1} \delta_{x_{j}}\right)\left(\mathrm{d} x_{m}\right)\left(\mu-\sum_{j=1}^{m-2} \delta_{x_{j}}\right)\left(\mathrm{d} x_{m-1}\right) \times \cdots \\
& \times\left(\mu-\delta_{x_{1}}\right)\left(\mathrm{d} x_{2}\right) \mu\left(\mathrm{d} x_{1}\right),
\end{aligned}
$$

where $\delta_{x}$ is the Dirac measure located at a point $x \in \mathbb{X}$. If $\mu$ is given as $\mu=\sum_{I} \delta_{x_{i}}$ for some countable index set $I$ and $x_{i} \in \mathbb{X}, i \in I$, then

$$
\int f \mathrm{~d} \mu^{(m)}=\sum_{i_{1}, \ldots, i_{m} \in I}^{\neq} f\left(x_{i_{1}}, \ldots, x_{i_{m}}\right),
$$

where $f$ is any nonnegative measurable function on $\mathbb{X}^{m}$ and where the superscript ' $\neq$ ' indicates that we sum over $m$-tuples of disjoint indices.

We will use the multivariate Mecke-formula (see, e.g. [10]),

$$
\begin{aligned}
\mathbb{E} \int h\left(\eta, x_{1}, \ldots, x_{m}\right) \eta^{(m)}\left(\mathrm{d}\left(x_{1}, \ldots, x_{m}\right)\right) \\
\quad=\mathbb{E} \int h\left(\eta+\delta_{x_{1}}+\cdots+\delta_{x_{m}}, x_{1}, \ldots, x_{m}\right) \lambda^{m}\left(\mathrm{~d}\left(x_{1}, \ldots, x_{m}\right)\right),
\end{aligned}
$$

which holds for all $h: N \times \mathbb{X}^{m} \rightarrow \mathbb{R}$ for which one (and then also the other) side makes sense.

For any integer $n \geq 1$ let $L_{s}^{1}\left(\lambda^{n}\right)$ denote the set of all measurable and symmetric functions $f: \mathbb{X}^{n} \rightarrow \mathbb{R}$ that are integrable with respect to $\lambda^{n}$. For $f \in L_{s}^{1}\left(\lambda^{n}\right)$ define the pathwise multiple Wiener-Itô integral by

$$
I_{n}(f):=\sum_{J \subset[n]}(-1)^{n-|J|} \iint f\left(x_{1}, \ldots, x_{n}\right) \eta^{(|J|)}\left(\mathrm{d} x_{J}\right) \lambda^{n-|J|}\left(\mathrm{d} x_{J^{c}}\right),
$$

where $[n]:=\{1, \ldots, n\}, J^{c}:=[n] \backslash J, x_{J}:=\left(x_{j}\right)_{j \in J}$, and where $|J|$ denotes the cardinality of $J$ (the inner integral is interpreted as $f\left(x_{1}, \ldots, x_{n}\right)$ in the case where $J=\varnothing$ ). By (2), $I_{n}(f)$ is a well-defined integrable random variable with $\mathbb{E} I_{n}(f)=0$. If $f \in L_{s}^{1}\left(\lambda^{n}\right) \cap L^{2}\left(\lambda^{n}\right)$, this pathwise definition coincides with the (classical) definition of the multiple Wiener-Itô integral for square integrable functions. (This fact can be derived, for instance, from [10, Equation (3.1)].) The multiple Wiener-Itô integral $I_{n}(f)$ for (symmetric) $f \in L^{2}\left(\lambda^{n}\right)$ is defined by an extension of the pathwise definition for $L^{1}$-functions to the space of all square 
integrable random variables. It also has mean zero and satisfies the orthogonality and isometry relations

$$
\mathbb{E} I_{m}(g) I_{n}(h)=\mathbf{1}_{\{m=n\}} n !\langle g, h\rangle_{n}, \quad m, n \geq 1,
$$

for all (symmetric) $g \in L^{2}\left(\lambda^{m}\right)$ and $h \in L^{2}\left(\lambda^{n}\right)$, where $\langle\cdot, \cdot\rangle_{n}$ denotes the scalar product in $L^{2}\left(\lambda^{n}\right)$.

For $x \in \mathbb{X}$ the difference operator $D_{x}$ is given as follows. For any measurable $F: N \rightarrow \mathbb{R}$ the function $D_{x} F$ on $N$ is defined by $D_{x} F(\mu):=F\left(\mu+\delta_{x}\right)-F(\mu), \mu \in N$. For $n \geq 2$ and $\left(x_{1}, \ldots, x_{n}\right) \in \mathbb{X}^{n}$ we define a function $D_{x_{1}, \ldots, x_{n}}^{n} F: N \rightarrow \mathbb{R}$ by an iterated application of the difference operator $D$, that is, inductively by

$$
D_{x_{1}, \ldots, x_{n}}^{n} F:=D_{x_{1}}^{1} D_{x_{2}, \ldots, x_{n}}^{n-1} F,
$$

where $D_{x}^{1}:=D_{x}$ and $D^{0} F:=F$. Under the assumption $\mathbb{E} F(\eta)^{2}<\infty$, it was proved in [10] that $D_{x_{1}, \ldots, x_{n}}^{n} F(\eta)$ is integrable for $\lambda^{n}$-almost every $\left(\lambda^{n}\right.$-a.e. $)\left(x_{1}, \ldots, x_{n}\right)$ and that

$$
T_{n} F\left(x_{1}, \ldots, x_{n}\right):=\mathbb{E} D_{x_{1}, \ldots, x_{n}}^{n} F(\eta), \quad\left(x_{1}, \ldots, x_{n}\right) \in \mathbb{X}^{n},
$$

defines a symmetric function in $L^{2}\left(\lambda^{n}\right)$. Moreover, we have the Wiener-Itô chaos expansion

$$
F(\eta)=\mathbb{E} F+\sum_{n=1}^{\infty} \frac{1}{n !} I_{n}\left(T_{n} F\right),
$$

where the series converges in $L^{2}(\mathbb{P})$. Hence (1) holds with $f_{n}=(1 / n !) T_{n} F$.

\section{Moments and cumulants}

Let $n \in \mathbb{N}$. A subpartition of $[n]$ is a family of disjoint and nonempty subsets of $[n]$, which we call blocks. A partition of $[n]$ is a subpartition $\sigma$ of $[n]$ such that $\bigcup_{J \in \sigma} J=[n]$. We denote by $\Pi_{n}$ (respectively $\Pi_{n}^{*}$ ) the system of all partitions (respectively subpartitions) of $[n]$. The cardinality of $\sigma \in \Pi_{n}^{*}$ (i.e. the number of blocks of $\sigma$ ) is denoted by $|\sigma|$, while the cardinality of $\bigcup_{J \in \sigma} J$ is denoted by $\|\sigma\|$. For any function $f: \mathbb{X}^{n} \rightarrow \mathbb{R}$ and $\sigma \in \Pi_{n}^{*}$ we define $f_{\sigma}: \mathbb{X}^{|\sigma|+n-\|\sigma\|} \rightarrow \mathbb{R}$ by identifying the arguments belonging to the same $J \in \sigma$. (The arguments $x_{1}, \ldots, x_{|\sigma|+n-\|\sigma\|}$ have to be inserted in the order of occurrence.) In the case $n=4$ and $\sigma=\{\{2,3\},\{4\}\}$, for instance, we have $f_{\sigma}\left(x_{1}, x_{2}, x_{3}\right)=f\left(x_{1}, x_{2}, x_{2}, x_{3}\right)$.

Consider $\ell, n_{1}, \ldots, n_{\ell} \in \mathbb{N}$. Define $n:=n_{1}+\cdots+n_{\ell}$ and

$$
J_{i}:=\left\{j: n_{1}+\cdots+n_{i-1}<j \leq n_{1}+\cdots+n_{i}\right\}, \quad i=1, \ldots, \ell .
$$

Let $\pi:=\left\{J_{i}: 1 \leq i \leq \ell\right\}$ and let $\Pi\left(n_{1}, \ldots, n_{\ell}\right) \subset \Pi_{n}$ (respectively $\left.\Pi^{*}\left(n_{1}, \ldots, n_{\ell}\right) \subset \Pi_{n}^{*}\right)$ denote the set of all $\sigma \in \Pi_{n}$ (respectively $\sigma \in \Pi_{n}^{*}$ ) with $\left|J \cap J^{\prime}\right| \leq 1$ for all $J \in \pi$ and all $J^{\prime} \in \sigma$. Let $\Pi_{\geq 2}\left(n_{1}, \ldots, n_{\ell}\right)$ (respectively $\left.\Pi_{=2}\left(n_{1}, \ldots, n_{\ell}\right)\right)$ denote the set of all $\sigma \in$ $\Pi\left(n_{1}, \ldots, n_{\ell}\right)$ with $|J| \geq 2$ (respectively $|J|=2$ ) for all $J \in \sigma$. It is instructive to visualize the pair $(\pi, \sigma)$ as a diagram with rows $J_{1}, \ldots, J_{\ell}$, where the elements in each $J \in \sigma$ are encircled by a closed curve; see [12, Chapter 4] for more details on such diagrams. Since the blocks of a $\sigma \in \Pi\left(n_{1}, \ldots, n_{\ell}\right)$ are not allowed to contain more than one entry from each row, the diagram $(\pi, \sigma)$ is called nonflat in [12]. Any $\sigma \in \Pi_{\geq 2}\left(n_{1}, \ldots, n_{\ell}\right)$ induces a partition $\sigma^{*} \in \Pi_{\ell}: \sigma^{*}$ is the finest partition of $[\ell]$ such that two numbers $i, j \in[\ell]$ are in the same block of $\sigma^{*}$ if $J_{i}$ and $J_{j}$ are both intersected by the same block of $\sigma$. Let $\tilde{\Pi}_{\geq 2}\left(n_{1}, \ldots, n_{\ell}\right)$ be the set of all $\sigma \in \Pi_{\geq 2}\left(n_{1}, \ldots, n_{\ell}\right)$ such that $\left|\sigma^{*}\right|=1$. 
The tensor product $\bigotimes_{i=1}^{\ell} f_{i}$ of functions $f_{i}: \mathbb{X}^{n_{i}} \rightarrow \mathbb{R}, i \in\{1, \ldots, \ell\}$, is the function from $\mathbb{X}^{n}$ to $\mathbb{R}$ which maps each $\left(x_{1}, \ldots, x_{n}\right)$ to $\prod_{i=1}^{n} f_{i}\left(x_{J_{i}}\right)$. In case that $f_{1}=\cdots=f_{\ell}=f$ we write $f^{\otimes \ell}$ instead of $\bigotimes_{i=1}^{\ell} f_{i}$.

The joint cumulant $\gamma\left(X_{1}, \ldots, X_{\ell}\right)$ of $\ell \geq 1$ random variables $X_{1}, \ldots, X_{\ell}$ is defined as

$$
\gamma\left(X_{1}, \ldots, X_{\ell}\right):=\left.(-\mathrm{i})^{\ell} \frac{\partial^{\ell}}{\partial z_{1} \cdots \partial z_{\ell}} \log \mathbb{E}\left[\exp \left[\mathrm{i}\left(z_{1} X_{1}+\cdots+z_{\ell} X_{\ell}\right)\right]\right]\right|_{z_{1}=\cdots=z_{\ell}=0},
$$

where $\mathrm{i}$ is the imaginary unit. This cumulant is well defined if $\prod_{j \in I} X_{j}$ is integrable for all $I \subset[\ell]$. The $\ell$ th cumulant of a single random variable $X$ is defined by $\gamma_{\ell}(X):=\gamma(X, \ldots, X)$, where $X$ appears $\ell$ times.

The following result generalizes [12, Corollary 7.4.1] and a consequence of [19] to the case of more general Poisson processes and integrands. In contrast to [12] and [19], we allow that the intensity measure has atoms. Moreover, we avoid the assumption in [12, Corollary 7.4.1] that the integrands are simple functions. While the results in [12] and [19] are derived via formulae for the Wiener-Itô chaos expansion of a product of multiple Wiener-Itô integrals, here we take a direct approach, which relies only on (2) and some combinatorial arguments.

Theorem 1. Let $f_{i} \in L_{s}^{1}\left(\lambda^{n_{i}}\right), i=1, \ldots, \ell$, where $\ell, n_{1}, \ldots, n_{\ell} \in \mathbb{N}$. Assume that

$$
\int\left(\bigotimes_{i=1}^{\ell}\left|f_{i}\right|\right)_{\sigma} \mathrm{d} \lambda^{|\sigma|}<\infty, \quad \sigma \in \Pi\left(n_{1}, \ldots, n_{\ell}\right) .
$$

Then

$$
\begin{gathered}
\mathbb{E} \prod_{i=1}^{\ell} I_{n_{i}}\left(f_{i}\right)=\sum_{\sigma \in \Pi_{\geq 2}\left(n_{1}, \ldots, n_{\ell}\right)} \int\left(\bigotimes_{i=1}^{\ell} f_{i}\right)_{\sigma} \mathrm{d} \lambda^{|\sigma|}, \\
\gamma\left(I_{n_{1}}\left(f_{1}\right), \ldots, I_{n_{\ell}}\left(f_{\ell}\right)\right)=\sum_{\sigma \in \tilde{\Pi}_{\geq 2}\left(n_{1}, \ldots, n_{\ell}\right)} \int\left(\bigotimes_{i=1}^{\ell} f_{i}\right)_{\sigma} \mathrm{d} \lambda^{|\sigma|} .
\end{gathered}
$$

Proof. We abbreviate $f:=\bigotimes_{i=1}^{\ell} f_{i}$. The definition (3) and Fubini's theorem imply that

$$
\begin{aligned}
\prod_{i=1}^{\ell} I_{n_{i}}\left(f_{i}\right)= & \sum_{I \subset[n]}(-1)^{n-|I|} \\
& \times \int \cdots \int f\left(x_{1}, \ldots, x_{n}\right) \\
& \times \eta^{\left(\left|I \cap J_{1}\right|\right)}\left(\mathrm{d} x_{I \cap J_{1}}\right) \cdots \eta^{\left(\left|I \cap J_{\ell}\right|\right)}\left(\mathrm{d} x_{I \cap J_{\ell}}\right) \lambda^{n-|I|}\left(\mathrm{d} x_{I^{c}}\right),
\end{aligned}
$$

where $I^{c}:=[n] \backslash I$, and where we use definition (6) of $J_{i}$. For fixed $I \subset[n]$ we may split the above integration according to $\sigma \in \Pi^{*}\left(n_{1}, \ldots, n_{\ell}\right)$, where $\bigcup_{J \in \sigma} J=I$. For any such $\sigma$ we integrate (i.e. sum) over those $\left(x_{1}, \ldots, x_{n}\right)$ satisfying $x_{i}=x_{j}$ whenever $i$ and $j$ belong to the same block of $\sigma$ but not otherwise. By (2) applied with $h\left(\eta, y_{1}, \ldots, y_{|\sigma|}\right)=f\left(x_{1}, \ldots, x_{m}\right)$ taking $x_{i}=y_{j}$ for $i$ in the $j$ th block of $\sigma$, the contribution of $\sigma$ to the expectation of the right-hand side of (10) is equal to $(-1)^{n-\|\sigma\|} \int f_{\sigma} \mathrm{d} \lambda^{|\sigma|+n-\|\sigma\|}$. Therefore,

$$
\mathbb{E} \prod_{i=1}^{\ell} I_{n_{i}}\left(f_{i}\right)=\sum_{\sigma \in \Pi^{*}\left(n_{1}, \ldots, n_{\ell}\right)}(-1)^{n-\|\sigma\|} \int f_{\sigma} \mathrm{d} \lambda^{|\sigma|+n-\|\sigma\|} .
$$


By (7), all of these integrals are finite. Take a $\sigma \in \Pi^{*}\left(n_{1}, \ldots, n_{\ell}\right)$ with $|J| \geq 2$ for all $J \in \sigma$ and consider the set $\Pi_{1}(\sigma)$ of all $\sigma_{1} \in \Pi^{*}\left(n_{1}, \ldots, n_{\ell}\right)$ such that $\sigma \subset \sigma_{1}$ and $|J| \leq 1$ for all $J \in \sigma_{1} \backslash \sigma$. (Note that $\sigma \in \Pi_{1}(\sigma)$.) Observe that $\int f_{\sigma_{1}} \mathrm{~d} \lambda^{\left|\sigma_{1}\right|+n-\left\|\sigma_{1}\right\|}=\int f_{\sigma} \mathrm{d} \lambda^{|\sigma|+n-\|\sigma\|}$ for all $\sigma_{1} \in \Pi_{1}(\sigma)$. Moreover, for $n-\|\sigma\| \geq 1$ we have

$$
\sum_{\sigma_{1} \in \Pi_{1}(\sigma)}(-1)^{n-\left\|\sigma_{1}\right\|}=0
$$

Since every $\tau \in \Pi^{*}\left(n_{1}, \ldots, n_{\ell}\right)$ has a unique $\sigma \in \Pi^{*}\left(n_{1}, \ldots, n_{\ell}\right)$ with $|J| \geq 2$ for all $J \in \sigma$ such that $\tau \in \Pi_{1}(\sigma)$, we can partition $\Pi^{*}\left(n_{1}, \ldots, n_{\ell}\right)$ into the sets $\Pi_{1}(\sigma), \sigma \in$ $\Pi^{*}\left(n_{1}, \ldots, n_{\ell}\right)$, with $|J| \geq 2$ for all $J \in \sigma$. As shown above, the sums over all $\sigma_{1} \in \Pi_{1}(\sigma)$ with $\|\sigma\|<n$ vanish and only the integrals related to partitions $\sigma \in \Pi_{\geq 2}\left(n_{1}, \ldots, n_{\ell}\right)$ remain. Therefore, (11) implies the asserted identity (1).

We now prove (9) by induction over $\ell$. Since $\gamma\left(I_{n_{1}}\left(f_{1}\right), I_{n_{2}}\left(f_{2}\right)\right)=\mathbb{E} I_{n_{1}}\left(f_{1}\right) I_{n_{2}}\left(f_{2}\right)$ and $\Pi_{\geq 2}\left(n_{1}, n_{2}\right)=\tilde{\Pi}_{\geq 2}\left(n_{1}, n_{2}\right)$, (9) is true for $\ell=2$; see (1). For $\ell \geq 3$, we obtain, by the inversion formula expressing the $\ell$ th moment in terms of lower-order cumulants (see, e.g. [12, Proposition 3.2.1]), (1), and the assumption of the induction,

$$
\begin{aligned}
& \gamma\left(I_{n_{1}}\left(f_{1}\right), \ldots, I_{n_{\ell}}\left(f_{\ell}\right)\right) \\
& =\mathbb{E} \prod_{j=1}^{\ell} I_{n_{j}}\left(f_{j}\right)-\sum_{\pi \in \Pi_{\ell},|\pi|>1} \prod_{J \in \pi} \gamma\left(\left(I_{n_{j}}\left(f_{j}\right)\right)_{j \in J}\right) \\
& =\sum_{\sigma \in \Pi_{\geq 2}\left(n_{1}, \ldots, n_{\ell}\right)} \int f_{\sigma} \mathrm{d} \lambda^{|\sigma|}-\sum_{\pi \in \Pi_{\ell},|\pi|>1} \prod_{J \in \pi} \sum_{\sigma_{J} \in \tilde{\Pi}_{\geq 2}(J)} \int\left(\bigotimes_{j \in J} f_{j}\right)_{\sigma_{J}} \mathrm{~d} \lambda^{\left|\sigma_{J}\right|} .
\end{aligned}
$$

Here, $\tilde{\Pi}_{\geq 2}(J)$ is defined in a similar way to $\tilde{\Pi}_{\geq 2}\left(n_{1}, \ldots, n_{\ell}\right)$. Now we use the fact that every partition $\sigma \in \Pi_{\geq 2}\left(n_{1}, \ldots, n_{\ell}\right)$ uniquely determines (in the obvious way) a partition $\pi=\sigma^{*} \in \Pi_{\ell}$ and a collection of partitions $\sigma_{J} \in \tilde{\Pi}_{\geq 2}(J), J \in \pi$, and vice versa. Combining this with Fubini's theorem, we have

$$
\begin{aligned}
\sum_{\pi \in \Pi_{\ell},|\pi|>1} \prod_{J \in \pi} \sum_{\sigma J \in \tilde{\Pi}_{\geq 2}(J)} \int\left(\bigotimes_{j \in J} f_{j}\right)_{\sigma_{J} \mathrm{~d} \lambda^{\left|\sigma_{J}\right|}} & =\sum_{\sigma \in \Pi_{\geq 2}\left(n_{1}, \ldots, n_{\ell}\right),\left|\sigma^{*}\right|>1} \int\left(\prod_{J \in \sigma^{*}} \bigotimes_{j \in J} f_{j}\right)_{\sigma} \mathrm{d} \lambda^{|\sigma|} \\
& =\sum_{\sigma \in \Pi_{\geq 2}\left(n_{1}, \ldots, n_{\ell}\right),\left|\sigma^{*}\right|>1} \int f_{\sigma} \mathrm{d} \lambda^{|\sigma|} .
\end{aligned}
$$

Hence, in (12) only the partitions $\sigma \in \Pi_{\geq 2}\left(n_{1}, \ldots, n_{\ell}\right)$ with $\left|\sigma^{*}\right|=1$ remain. In our notation, these are exactly the partitions in $\tilde{\Pi}_{\geq 2}\left(n_{1}, \ldots, n_{\ell}\right)$.

Remark 1. The assumption (7) is obviously satisfied if $f_{i}$ is bounded and $\lambda^{n_{i}}\left(\left\{f_{i} \neq 0\right\}\right)<\infty$ for $i=1, \ldots, \ell$, which is the case for our examples in Section 6 , below. But the assumption also holds under the following conditions. Consider, as in Theorem 1, measurable functions $f_{i}: \mathbb{X}^{n_{i}} \rightarrow \mathbb{R}$, for $i=1, \ldots, \ell$. Assume for any $i$ that $\left\{f_{i} \neq 0\right\} \subset B^{n_{i}}$, where $B \in \mathcal{X}$ satisfies $\lambda(B)<\infty$. For any $i$, if $f_{i} \in L^{\ell}\left(\lambda^{n_{i}}\right)$ then $f_{i} \in L^{1}\left(\lambda^{n_{i}}\right)$ and (7) holds. The second assertion follows from the multivariate version of Hölder's inequality. In fact, if $\sigma \in \Pi\left(n_{1}, \ldots, n_{\ell}\right)$ then

$$
\left(\int\left(\bigotimes_{i=1}^{\ell}\left|f_{i}\right|\right)_{\sigma} \mathrm{d} \lambda^{|\sigma|}\right)^{\ell} \leq \lambda(B)^{|\sigma|-n_{1}} \int\left|f_{1}\right|^{\ell} \mathrm{d} \lambda^{n_{1}} \cdots \lambda(B)^{|\sigma|-n_{\ell}} \int\left|f_{\ell}\right|^{\ell} \mathrm{d} \lambda^{n_{\ell}} .
$$


Another sufficient condition for the assumptions of Theorem 1 is the existence of a function $g \in L^{1}(\lambda) \cap L^{\ell}(\lambda)$ such that $\left|f_{i}\right| \leq g^{\otimes n_{i}}$ for any $i$. In this case, we have for $\sigma \in \Pi\left(n_{1}, \ldots, n_{\ell}\right)$ that

$$
\int\left(\bigotimes_{i=1}^{\ell}\left|f_{i}\right|\right)_{\sigma} \mathrm{d} \lambda^{|\sigma|} \leq \int g^{\otimes i_{1}} \mathrm{~d} \lambda \cdots \int g^{\otimes i_{|\sigma|}} \mathrm{d} \lambda
$$

where $i_{1}, \ldots, i_{|\sigma|} \leq \ell$ are the cardinalities of the blocks of $\sigma$.

Example 1. Let $f \in L_{s}^{1}\left(\lambda^{2}\right)$ and consider Theorem 1 in the case $\ell=2, n_{1}=n_{2}=2$, and $f_{1}=f_{2}=f$. Then it is easy to see that assumption (7) requires $f \in L^{2}\left(\lambda^{2}\right)$ and

$$
\int\left[\int\left|f\left(x_{1}, x_{2}\right)\right| \lambda\left(\mathrm{d} x_{2}\right)\right]^{2} \lambda\left(\mathrm{d} x_{1}\right)<\infty .
$$

Formula (1) boils down to the isometry relation $\mathbb{E} I_{2}(f)^{2}=2\langle f, f\rangle_{2}$. This shows that assumption (7) is not necessary for (1).

Example 2. Let $f \in L_{s}^{1}\left(\lambda^{2}\right)$ and $g \in L^{1}(\lambda)$ and consider Theorem 1 in the case $\ell=3$, $n_{1}=n_{2}=2, f_{1}=f_{2}=f, n_{3}=1$, and $f_{3}=g$. Assumption (7) then requires $f$ to satisfy the same integrability conditions as in Example 1, as well as

$$
\begin{gathered}
\int\left(\left|f\left(x_{1}, x_{2}\right)\right|+f\left(x_{1}, x_{2}\right)^{2}\right)\left|g\left(x_{1}\right)\right| \lambda^{2}\left(\mathrm{~d}\left(x_{1}, x_{2}\right)\right)<\infty, \\
\int\left|f\left(x_{1}, x_{2}\right) f\left(x_{2}, x_{3}\right)\right|\left(\left|g\left(x_{1}\right)\right|+\left|g\left(x_{2}\right)\right|\right) \lambda^{3}\left(\mathrm{~d}\left(x_{1}, x_{2}, x_{3}\right)\right)<\infty .
\end{gathered}
$$

Equation (1) means that

$$
\mathbb{E}\left[I_{2}(f)^{2} I_{1}(g)\right]=4 \int f\left(x_{1}, x_{2}\right)^{2} g\left(x_{1}\right) \lambda^{3}\left(\mathrm{~d}\left(x_{1}, x_{2}\right)\right) .
$$

Note that we do not need to assume that $g$ is square-integrable with respect to $\lambda$.

Corollary 1. Let $f_{n} \in L_{s}^{1}\left(\lambda^{n}\right), n \in \mathbb{N}$, and let $\ell \in \mathbb{N}$ and assume that

$$
\int\left(\bigotimes_{i=1}^{\ell}\left|f_{n_{i}}\right|\right)_{\sigma} \mathrm{d} \lambda^{|\sigma|}<\infty, \quad \sigma \in \Pi\left(n_{1}, \ldots, n_{\ell}\right), n_{1}, \ldots, n_{\ell} \in \mathbb{N} .
$$

Assume further that $\mathbb{E}\left(\sum_{n=1}^{\infty}\left|I_{n}\left(f_{n}\right)\right|\right)^{\ell}<\infty$. Then the $\ell$ th moment and the $\ell$ th cumulant of $F:=\sum_{n=1}^{\infty} I_{n}\left(f_{n}\right)$ are given by

$$
\begin{aligned}
& \mathbb{E} F^{\ell}=\sum_{n_{1}, \ldots, n_{\ell} \in \mathbb{N}} \sum_{\sigma \in \Pi_{\geq 2}\left(n_{1}, \ldots, n_{\ell}\right)} \int\left(\bigotimes_{i=1}^{\ell} f_{n_{i}}\right)_{\sigma} \mathrm{d} \lambda^{|\sigma|}, \\
& \gamma_{\ell}(F)=\sum_{n_{1}, \ldots, n_{\ell} \in \mathbb{N}} \sum_{\sigma \in \tilde{\Pi}_{\geq 2}\left(n_{1}, \ldots, n_{\ell}\right)} \int\left(\bigotimes_{i=1}^{\ell} f_{n_{i}}\right)_{\sigma} \mathrm{d} \lambda^{|\sigma|} .
\end{aligned}
$$

Proof. Let $m \in \mathbb{N}$ and $F_{m}:=I_{1}\left(f_{1}\right)+\cdots+I_{m}\left(f_{m}\right)$. Expanding $\left(F_{m}\right)^{\ell}$ and using (1) gives

$$
\begin{aligned}
\mathbb{E} F_{m}^{\ell} & =\sum_{n_{1}, \ldots, n_{\ell}=1}^{m} \mathbb{E}\left(I_{n_{1}}\left(f_{n_{1}}\right) \cdots I_{n_{l}}\left(f_{n_{l}}\right)\right) \\
& =\sum_{n_{1}, \ldots, n_{\ell}=1}^{m} \sum_{\sigma \in \Pi_{\geq 2}\left(n_{1}, \ldots, n_{\ell}\right)} \int\left(\bigotimes_{i=1}^{\ell} f_{n_{i}}\right)_{\sigma} \mathrm{d} \lambda^{|\sigma|} .
\end{aligned}
$$


The assumption that $\mathbb{E}\left(\sum_{n=1}^{\infty}\left|I_{n}\left(f_{n}\right)\right|\right)^{\ell}<\infty$ and dominated convergence imply (14) for the infinite case. By the multilinearity of joint cumulants and (9),

$$
\gamma_{\ell}\left(F_{m}\right)=\sum_{n_{1}, \ldots, n_{\ell}=1}^{m} \sum_{\sigma \in \tilde{\Pi}_{\geq 2}\left(n_{1}, \ldots, n_{\ell}\right)} \int\left(\bigotimes_{i=1}^{\ell} f_{n_{i}}\right)_{\sigma} \mathrm{d} \lambda^{|\sigma|} .
$$

Since $\gamma_{\ell}\left(F_{m}\right)$ is a polynomial in the moments $\mathbb{E} F_{m}^{j}, j \in\{1, \ldots, \ell\}$, whose coefficients are independent of $m$ (or $F_{m}$ ), we can again use dominated convergence to conclude the result (15) for the infinite case.

Remark 2. If in Corollary 1 the number of nonvanishing functions $f_{n} \not \equiv 0$ is finite, then the assumption $\mathbb{E}\left(\sum_{n=1}^{\infty}\left|I_{n}\left(f_{n}\right)\right|\right)^{\ell}<\infty$ is implied by (13).

Let $f \in L^{2}\left(\lambda^{2}\right)$ be given by $f\left(x_{1}, x_{2}\right):=\mathbf{1}_{\left\{x_{1} \in B, x_{2} \in B\right\}}$, where $\lambda(B)<\infty$. By (3),

$$
I_{2}(f)=\eta(B)(\eta(B)-1)-2 \eta(B) \lambda(B)+\lambda(B)^{2} .
$$

A straightforward calculation shows that $\mathbb{E} \exp \left(s I_{2}(f)\right)=\infty$ for all $s>0$, whenever $\lambda(B)>0$. Our next result shows that this is a quite general property of Poisson functionals.

Corollary 2. Let $F=\sum_{n=1}^{\infty} I_{n}\left(f_{n}\right)$ with $f_{n} \in L_{s}^{1}\left(\lambda^{n}\right), n \in \mathbb{N}$, and assume that

$$
\int\left(\bigotimes_{i=1}^{\ell}\left|f_{n_{i}}\right|\right)_{\sigma} \mathrm{d} \lambda^{|\sigma|}<\infty \text { for all } \sigma \in \Pi\left(n_{1}, \ldots, n_{\ell}\right) \text { with } n_{1}, \ldots, n_{\ell} \in \mathbb{N}
$$

and that $\mathbb{E}\left(\sum_{n=1}^{\infty}\left|I_{n}\left(f_{n}\right)\right|\right)^{\ell}<\infty$ for all $\ell \in \mathbb{N}$. In addition, suppose that $f_{n} \geq 0$ for all $n \in \mathbb{N}$ and that there is an $n_{0} \geq 2$ with $\left\|f_{n_{0}}\right\|_{n_{0}}>0$. Then $\mathbb{E} \exp (s F)=\infty$ for all $s>0$.

Proof. The idea of the proof is to show that the number of partitions we sum over in (14) and (15) is rapidly increasing in $\ell$. For $\ell \in \mathbb{N}$ with $6 \mid \ell$ (i.e. $\ell$ a multiple of 6 ) let $\Pi_{\ell}^{(2)} \subset$ $\tilde{\Pi}_{\geq 2}(2, \ldots, 2)$ be the set of partitions of $[2 \ell]$ that can be constructed in the following way. First, the odd numbers in $[2 \ell]$ are partitioned into blocks of size 6 . Then we form $\ell / 6-1$ blocks of size 2 from the even numbers of $[2 \ell]$ such that all partitions from $\Pi_{\geq 2}(2, \ldots, 2)$ that contain the subpartition constructed so far must belong to $\tilde{\Pi}_{\geq 2}(2, \ldots, 2)$. Finally, we

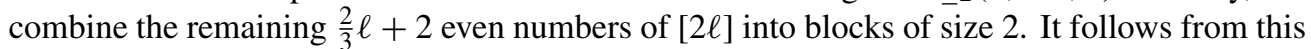
construction and a short computation that

$$
\left|\Pi_{\ell}^{(2)}\right| \geq \frac{\ell !}{(\ell / 6) !(6 !)^{\ell / 6}} \frac{(2 \ell / 3+2) !}{(\ell / 3+1) ! 2^{\ell / 3+1}} \geq \frac{\ell !(\ell / 6) !}{(6 !)^{\ell}} .
$$

Note that we do not take into account here the different possibilities of forming the first $\ell / 6-1$ blocks from the even numbers of $[2 \ell]$. The previous inequality implies that

$$
\left|\Pi_{\geq 2}\left(n_{1}, \ldots, n_{\ell}\right)\right| \geq\left|\tilde{\Pi}_{\geq 2}\left(n_{1}, \ldots, n_{\ell}\right)\right| \geq\left|\Pi_{\ell}^{(2)}\right| \geq \frac{\ell !(\ell / 6) !}{(6 !)^{\ell}}
$$

if $n_{1} \geq 2, \ldots, n_{\ell} \geq 2$ and $\ell \in \mathbb{N}$ is such that $6 \mid \ell$.

Each $\tilde{\sigma} \in \Pi_{\ell}^{(2)}$ with $\ell \in \mathbb{N}$ and $6 \mid \ell$ induces, for every $k \geq 2$, a partition $\sigma \in \tilde{\Pi}_{\geq 2}(k, \ldots, k)$ of $[k \ell]$ in the following way. First, we partition $\{1+j k: j=0, \ldots, \ell-1\}$ as the odd numbers in $\tilde{\sigma}$ and then, for each $i=2, \ldots, k$, the sets $\{i+j k: j=0, \ldots, \ell-1\}$ as the even numbers in $\tilde{\sigma}$. We denote the set of these partitions by $\Pi_{\ell}^{(k)}$. 
Owing to the assumptions that $\left\|f_{n_{0}}\right\|_{n_{0}}>0$ and $f_{n_{0}}$ is nonnegative, and the structure of $\Pi_{\ell}^{\left(n_{0}\right)}$, there must be a constant $c>0$ such that

$$
\int\left(f_{n_{0}}^{\otimes \ell}\right)_{\sigma} \mathrm{d} \lambda^{|\sigma|} \geq c^{\ell}
$$

for all $\ell \in \mathbb{N}$ with $6 \mid \ell$ and $\sigma \in \Pi_{\ell}^{\left(n_{0}\right)}$. It follows from Corollary 1 and the estimates (16) and (17) that

$$
\mathbb{E} F^{\ell} \geq \frac{\ell !(\ell / 6) !}{(6 !)^{\ell}} c^{\ell} \quad \text { and } \quad \gamma_{\ell}(F) \geq \frac{\ell !(\ell / 6) !}{(6 !)^{\ell}} c^{\ell}
$$

for all $\ell \in \mathbb{N}$ with $6 \mid \ell$. This implies that $\mathbb{E} \exp (s F)=\infty$ for all $s>0$.

\section{Asymptotic behaviour of moments and cumulants}

In this section we consider Poisson processes $\eta_{t}$ with intensity measures $\lambda_{t}:=t \lambda, t>0$. We are interested in functionals of $\eta_{t}$ that can be represented as $F_{t}=g(t) \sum f\left(x_{1}, \ldots, x_{m}\right)$ with the sum running over all $m$-tuples of distinct points of $\eta_{t}$ for some integer $m \geq 1$. This setting is taken from [16, Section 5], where a central limit theorem for $F_{t}$ as $t \rightarrow \infty$ is derived. We generalize this to a multivariate setting and investigate the asymptotic behaviour of such functionals as $t \rightarrow \infty$. More formally, fix $\ell \geq 1$ and, for $i=1, \ldots, \ell$, let $m_{i} \in \mathbb{N}$, $f^{(i)} \in L_{s}^{1}\left(\lambda^{m_{i}}\right)$, and $g_{i}:(0, \infty) \rightarrow \mathbb{R}$ such that $g_{i}(t) \neq 0$ for all (or at least for all sufficiently large) $t>0$. Now, define

$$
F_{t}^{(i)}:=g_{i}(t) \int f^{(i)}\left(x_{1}, \ldots, x_{m_{i}}\right) \eta_{t}^{\left(m_{i}\right)}\left(\mathrm{d}\left(x_{1}, \ldots, x_{m_{i}}\right)\right), \quad t>0 .
$$

By (2), we have

$$
\mathbb{E} F_{t}^{(i)}=g_{i}(t) t^{m_{i}} \int f^{(i)}\left(x_{1}, \ldots, x_{m_{i}}\right) \lambda^{m_{i}}\left(\mathrm{~d}\left(x_{1}, \ldots, x_{m_{i}}\right)\right) .
$$

For $n=1, \ldots, m_{i}$, define

$$
f_{n}^{(i)}\left(x_{1}, \ldots, x_{n}\right):=\left(\begin{array}{c}
m_{i} \\
n
\end{array}\right) \int f^{(i)}\left(x_{1}, \ldots, x_{n}, y_{1}, \ldots, y_{m_{i}-n}\right) \lambda^{m_{i}-n}\left(\mathrm{~d}\left(y_{1}, \ldots, y_{m_{i}-n}\right)\right)
$$

and denote by $I_{n, t}$ the $n$-fold Wiener-Itô integral with respect to $\eta_{t}$. We claim that, $\mathbb{P}$-almost surely, $F_{t}^{(i)}$ can be written as

$$
F_{t}^{(i)}=\mathbb{E} F_{t}^{(i)}+g_{i}(t) \sum_{n=1}^{m_{i}} t^{m_{i}-n} I_{n, t}\left(f_{n}^{(i)}\right) .
$$

Indeed, if $f_{n}^{(i)} \in L^{2}\left(\lambda^{n}\right)$ for all $n \leq m_{i}$ then $F_{t}^{(i)}$ is square-integrable and (20) is just a special case of the chaos expansion (5), cf. [16, Lemma 3.5]. The $L^{1}$-version can be derived by approximation or by a direct calculation (just substitute (19) into (3) and observe that all resulting terms cancel out, except the integral representation $(18)$ of $F_{t}^{(i)}$ ).

Write $\|\cdot\|_{n}$ for the norm and $\langle\cdot, \cdot\rangle_{n}$ for the inner product in $L^{2}\left(\lambda^{n}\right)$, and assume again that $f_{n}^{(i)} \in L^{2}\left(\lambda^{n}\right)$ for $n \leq m_{i}$. Equations (4) and (20) imply that

$$
\operatorname{var}\left[F_{t}^{(i)}\right]=g_{i}(t)^{2} \sum_{n=1}^{m_{i}} t^{2 m_{i}-2 n} n ! \int\left(f_{n}^{(i)}\right)^{2} \mathrm{~d} \lambda_{t}^{n}=g_{i}(t)^{2} \sum_{n=1}^{m_{i}} t^{2 m_{i}-n} n !\left\|f_{n}^{(i)}\right\|_{n}^{2}
$$


and

$$
\operatorname{cov}\left[F_{t}^{(i)}, F_{t}^{(j)}\right]=g_{i}(t) g_{j}(t) \sum_{n=1}^{\min \left\{m_{i}, m_{j}\right\}} t^{m_{i}+m_{j}-n} n !\left\langle f_{n}^{(i)}, f_{n}^{(j)}\right\rangle_{n} .
$$

The variances, covariances, mixed moments, and cumulants of higher order show the following asymptotic behaviour as $t \rightarrow \infty$.

Theorem 2. Assume that $\int\left(\bigotimes_{i=1}^{\ell}\left|f^{(i)}\right|\right)_{\sigma} \mathrm{d} \lambda^{|\sigma|}<\infty$ for all $\sigma \in \Pi\left(m_{1}, \ldots, m_{\ell}\right)$. Then

$$
\lim _{t \rightarrow \infty} \mathbb{E} \prod_{i=1}^{\ell} \frac{F_{t}^{(i)}-\mathbb{E} F_{t}^{(i)}}{g_{i}(t) t^{m_{i}-1 / 2}}=\sum_{\sigma \in \Pi_{=2}(1, \ldots, 1)} \int\left(\bigotimes_{i=1}^{\ell} f_{1}^{(i)}\right)_{\sigma} \mathrm{d} \lambda^{|\sigma|}
$$

and

$$
\lim _{t \rightarrow \infty} \frac{\gamma\left(F_{t}^{(1)}-\mathbb{E} F_{t}^{(1)}, \ldots, F_{t}^{(\ell)}-\mathbb{E} F_{t}^{(\ell)}\right)}{\prod_{i=1}^{\ell} g_{i}(t) t^{m_{i}-1 / 2}}=0, \quad \ell \geq 3 .
$$

Remark 3. Note that the right-hand side of (22) vanishes for odd $\ell$. Moreover, $\gamma\left(F_{t}^{(1)}-\right.$ $\left.\mathbb{E} F_{t}^{(1)}\right)=0$ and, for $\ell=2$, the left-hand side of (23) coincides with that of (22) and is equal to $\left\langle f_{1}^{(1)}, f_{1}^{(2)}\right\rangle_{1}$.

Proof of Theorem 2. We can assume without loss of generality that $g_{i}(t) \equiv 1$. Due to the special structure of $F_{t}^{(i)}$ and the kernels of its chaos expansion, the integrability assumptions on $\bigotimes_{i=1}^{\ell} f^{(i)}$ imply that $\int\left(\bigotimes_{i=1}^{\ell}\left|f_{n_{i}}^{(i)}\right|\right)_{\sigma} \mathrm{d} \lambda^{|\sigma|}<\infty$ for all $\sigma \in \Pi\left(n_{1}, \ldots, n_{\ell}\right)$ and $1 \leq n_{i} \leq$ $m_{i}, i=1, \ldots, \ell$. The latter is condition (13) for the functions $f_{n_{i}}^{(i)}$ in (20) and allows us to apply Theorem 1 , which yields

$$
\begin{aligned}
\mathbb{E} \prod_{i=1}^{\ell}\left(F_{t}^{(i)}-\mathbb{E} F_{t}^{(i)}\right) & =\sum_{1 \leq n_{1} \leq m_{1}, \ldots, 1 \leq n_{\ell} \leq m_{\ell}} \mathbb{E} \prod_{i=1}^{\ell} t^{m_{i}-n_{i}} I_{n_{i}, t}\left(f_{n_{i}}^{(i)}\right) \\
& =\sum_{1 \leq n_{1} \leq m_{1}, \ldots, 1 \leq n_{\ell} \leq m_{\ell}} \sum_{\sigma \in \Pi_{\geq 2}\left(n_{1}, \ldots, n_{\ell}\right)} \int\left(\bigotimes_{i=1}^{\ell} t^{m_{i}-n_{i}} f_{n_{i}}^{(i)}\right)_{\sigma} \mathrm{d} \lambda_{t}^{|\sigma|} .
\end{aligned}
$$

On the right-hand side, each summand has order $t^{\sum\left(m_{i}-n_{i}\right)+|\sigma|}$. Because of $|\sigma| \leq\left\lfloor\left(\sum n_{i}\right) / 2\right\rfloor$ and $\sum n_{i} \geq \ell$, we have $\sum\left(m_{i}-n_{i}\right)+|\sigma| \leq \sum m_{i}-\left\lceil\left(\sum n_{i}\right) / 2\right\rceil \leq \sum m_{i}-\lceil\ell / 2\rceil$, so that the maximal order is at most $t^{\sum m_{i}-\lceil\ell / 2\rceil}$. For even $\ell$ this is obtained if and only if $n_{1}=\cdots=$ $n_{\ell}=1$, and the partition $\sigma$ satisfies $|J|=2$ for all $J \in \sigma$. Exactly these summands remain as $t \rightarrow \infty$ since they have the same order as the denominator in (22); other summands vanish as $t \rightarrow \infty$. If $\ell$ is odd, the numerator has at most order $t^{\sum m_{i}-(\ell+1) / 2}$ (in fact, the order is attained) and the denominator has order $t^{\sum m_{i}-\ell / 2}$ so that the expression vanishes in the limit.

For the cumulant $\gamma\left(F_{t}^{(1)}-\mathbb{E} F_{t}^{(1)}, \ldots, F_{t}^{(\ell)}-\mathbb{E} F_{t}^{(\ell)}\right), \ell \geq 3$, we obtain by Theorem 1 the expression in the second line of (24), where this time the inner sum only runs over all partitions $\sigma \in \tilde{\Pi}_{\geq 2}\left(n_{1}, \ldots, n_{\ell}\right)$. Since $\tilde{\Pi}_{\geq 2}(1, \ldots, 1) \cap \Pi_{=2}(1, \ldots, 1)=\varnothing$ for $\ell \geq 3$, all summands have a lower order than the denominator in (23) and vanish as $t \rightarrow \infty$.

In the next result we take $F_{t}^{(1)}=\cdots=F_{t}^{(\ell)}=F_{t}$ with

$$
F_{t}:=g(t) \int f\left(x_{1}, \ldots, x_{m}\right) \eta_{t}^{(m)}\left(\mathrm{d}\left(x_{1}, \ldots, x_{m}\right)\right), \quad t>0,
$$


as in (18), where $g_{1}=\cdots=g_{\ell}=g, f^{(1)}=\cdots=f^{(\ell)}=f$, and $m_{1}=\cdots=m_{\ell}=m$. Since $\Pi_{=2}(1, \ldots, 1)$ has cardinality

$$
(\ell-1) ! !:=(\ell-1)(\ell-3) \cdots 3 \cdot 1
$$

for even $\ell \geq 2$, Theorem 2 implies the following result.

Corollary 3. Assume that $\int\left(|f|^{\otimes \ell}\right)_{\sigma} \mathrm{d} \lambda^{|\sigma|}<\infty$ for all $\sigma \in \Pi(m, \ldots, m)$ and that $\left\|f_{1}\right\|_{1}>0$. Then

$$
\lim _{t \rightarrow \infty} \frac{\mathbb{E}\left(F_{t}-\mathbb{E} F_{t}\right)^{\ell}}{\left(\operatorname{var}\left[F_{t}\right]\right)^{\ell / 2}}= \begin{cases}(\ell-1) ! ! & \text { if } \ell \text { is even }, \\ 0 & \text { if } \ell \text { is odd }\end{cases}
$$

and

$$
\lim _{t \rightarrow \infty} \gamma_{\ell}\left(\frac{F_{t}-\mathbb{E} F_{t}}{\sqrt{\operatorname{var}\left[F_{t}\right]}}\right)= \begin{cases}1 & \text { if } \ell=2 \\ 0 & \text { if } \ell \neq 2\end{cases}
$$

\section{Central limit theorems}

In what follows, we assume the same setting as in Section 4. More precisely, fix $\ell \in \mathbb{N}$, let $F_{t}^{(1)}, \ldots, F_{t}^{(\ell)}$ be defined as in (18), and assume for each $i \leq \ell$ that $f_{n}^{(i)} \in L^{2}\left(\lambda^{n}\right)$ for $n \leq m_{i}$. We shall at first show how the results of Section 4 lead to a multivariate central limit theorem via the method of moments. Let us define

$$
\hat{F}_{t}^{(i)}:=g_{i}(t)^{-1} t^{-\left(m_{i}-1 / 2\right)}\left(F_{t}^{(i)}-\mathbb{E} F_{t}^{(i)}\right)
$$

and note from (20) that

$$
\hat{F}_{t}^{(i)}=t^{1 / 2} \sum_{n=1}^{m_{i}} t^{-n} I_{n, t}\left(f_{n}^{(i)}\right) .
$$

Furthermore, by (21), we have the asymptotic covariances

$$
C_{i j}:=\lim _{t \rightarrow \infty} \operatorname{cov}\left[\hat{F}_{t}^{(i)}, \hat{F}_{t}^{(j)}\right]=\left\langle f_{1}^{(i)}, f_{1}^{(j)}\right\rangle_{1}=\int f_{1}^{(i)}(x) f_{1}^{(j)}(x) \lambda(\mathrm{d} x), \quad i, j \in\{1, \ldots, \ell\} .
$$

Proposition 1. Let $N$ be an $\ell$-dimensional centred Gaussian random vector with covariance matrix $\left(C_{i j}\right)_{i, j=1, \ldots, \ell}$. Assume that $\int\left(\otimes_{j=1}^{k}\left|f^{\left(i_{j}\right)}\right|\right)_{\sigma} \mathrm{d} \lambda^{|\sigma|}<\infty$ for all $k \in N, i_{1}, \ldots, i_{k} \in$ $\{1, \ldots, \ell\}$, and $\sigma \in \Pi\left(m_{i_{1}}, \ldots, m_{i_{k}}\right)$. Then $\left(\hat{F}_{t}^{(1)}, \ldots, \hat{F}_{t}^{(\ell)}\right)$ converges in distribution to $N$.

Proof. Observe first that $\gamma\left(\hat{F}_{t}^{(i)}\right)=\mathbb{E} \hat{F}_{t}^{(i)}=0$ for $1 \leq i \leq \ell$ and

$$
\gamma\left(\hat{F}_{t}^{(i)}, \hat{F}_{t}^{(j)}\right)=\operatorname{cov}\left[\hat{F}_{t}^{(i)}, \hat{F}_{t}^{(j)}\right] \rightarrow C_{i j} \quad \text { as } t \rightarrow \infty,
$$

for any $1 \leq i$ and $j \leq \ell$. Now fix integers $k \geq 3$ and $1 \leq i_{1} \leq \cdots \leq i_{k} \leq \ell$, and consider the joint cumulant $\gamma\left(\hat{F}_{t}^{\left(i_{1}\right)}, \ldots, \hat{F}_{t}^{\left(i_{k}\right)}\right)$. By homogeneity and $(25)$ it follows that

$$
\gamma\left(\hat{F}_{t}^{\left(i_{1}\right)}, \ldots, \hat{F}_{t}^{\left(i_{k}\right)}\right)=\frac{\gamma\left(F_{t}^{\left(i_{1}\right)}-\mathbb{E} F_{t}^{\left(i_{1}\right)}, \ldots, F_{t}^{\left(i_{k}\right)}-\mathbb{E} F_{t}^{\left(i_{k}\right)}\right)}{\prod_{j=1}^{k} g_{i_{j}}(t) t^{m_{i_{j}}-1 / 2}} ;
$$

whence, Theorem 2 implies that $\gamma\left(\hat{F}_{t}^{\left(i_{1}\right)}, \ldots, \hat{F}_{t}^{\left(i_{k}\right)}\right) \rightarrow 0$ as $t \rightarrow \infty$. The method of moments (or cumulants) now yields the multivariate limit theorem; see [2, p. 352]. In the univariate case the conclusion can also be directly drawn from Corollary 3. 
We now turn to a quantitative version of the multivariate central limit theorem. We measure the distance between two $\ell$-dimensional random vectors $X$ and $Y$ by

$$
d_{3}(X, Y):=\sup _{g \in \mathscr{H}}|\mathbb{E} g(X)-\mathbb{E} g(Y)|,
$$

where $\mathscr{H}$ is the set of all functions $h \in C^{3}\left(\mathbb{R}^{\ell}\right)$ that satisfy

$$
\max _{1 \leq i_{1} \leq i_{2} \leq \ell} \sup _{x \in \mathbb{R}^{\ell}}\left|\frac{\partial^{2} h(x)}{\partial x_{i_{1}} \partial x_{i_{2}}}\right| \leq 1, \quad \max _{1 \leq i_{1} \leq i_{2} \leq i_{3} \leq \ell} \sup _{x \in \mathbb{R}^{\ell}}\left|\frac{\partial^{3} h(x)}{\partial x_{i_{1}} \partial x_{i_{2}} \partial x_{i_{3}}}\right| \leq 1 .
$$

Note that convergence under the (pseudo-) metric $d_{3}$ implies convergence in distribution. In [13], bounds are derived for the $d_{3}$-distance to the multivariate normal, along with similar bounds using a similarly defined $d_{2}$-distance. We work with the result for the $d_{3}$-distance since the covariance matrix of the Gaussian random vector is allowed to be only positive semidefinite (this means that some linear combinations of the components of the limiting random vector may be constant). A nontrivial example for such a degenerate situation can be found in [4]. The multivariate normal approximation of Poisson $U$-statistics in the $d_{2}$-distance has been considered in [11].

In contrast to the univariate results for the Wasserstein distance discussed in Section 1, we can derive a multivariate result only for the $d_{3}$-metric since the underlying result in [13] is based on that distance. This is caused by the fact that the approaches used in [13] for the multivariate normal approximation, namely an interpolation technique and the multivariate Stein's method, require a higher degree of smoothness for the test functions.

We are now ready to state the Berry-Esseen-type inequality.

Theorem 3. Let $N$ be an $\ell$-dimensional centered Gaussian random vector with covariance matrix $\left(C_{i j}\right)_{i, j=1, \ldots, \ell}$. Assume that $\int\left|f_{1}^{(i)}\right|^{3} \mathrm{~d} \lambda<\infty$ for every $i \in\{1, \ldots, \ell\}$. Then there is a constant $\tilde{c}>0$ such that

$$
d_{3}\left(\left(\hat{F}_{t}^{(1)}, \ldots, \hat{F}_{t}^{(\ell)}\right), N\right) \leq \tilde{c} t^{-1 / 2}, \quad t \geq 1 .
$$

Remark 4. For $\ell=1$ it is possible to replace $d_{3}$ in Theorem 3 by the classical Wasserstein distance $d_{\mathrm{W}}$ and obtain $d_{\mathrm{W}}\left(\hat{F}_{t}, N\right) \leq c t^{-1 / 2}$, where $N$ is a standard Gaussian random variable with variance $\left\|f_{1}\right\|_{1}^{2}$ and $c$ is a constant (see [9, Theorem 7.3] or [16, Theorem 5.2] for a different rescaling). If $\left\|f_{1}\right\|_{1}=0$, this implies convergence in distribution to the constant random variable $N \equiv 0$. In this situation [9, Theorem 7.3] yields convergence in distribution to a higher-order Wiener-Itô integral with respect to a Gaussian random measure after a suitable (different) scaling.

Remark 5. Theorem 3 also holds if $F_{t}^{(1)}, \ldots, F_{t}^{(\ell)}$ are finite sums of random variables of the type in (18). In fact, under some additional conditions, any Poisson functional with finite Wiener-Itô chaos expansion can be represented in such a way; see [16].

We prepare for the proof of Theorem 3 by stating and proving the following lemma.

Lemma 1. Let $X$ and $Y$ be $\ell$-dimensional random vectors with $\mathbb{E} X=\mathbb{E} Y$ and Euclidean norms $\|X\|$ and $\|Y\|$ such that $\mathbb{E}\|X\|^{2}<\infty$ and $\mathbb{E}\|Y\|^{2}<\infty$. Then we have

$$
d_{3}(X, Y) \leq \ell \sqrt{\mathbb{E}\|X\|^{2}+\mathbb{E}\|Y\|^{2}} \sqrt{\mathbb{E}\|X-Y\|^{2}} .
$$

Proof. For $h \in \mathcal{H}, X=\left(X_{1}, \ldots, X_{\ell}\right)$, and $Y=\left(Y_{1}, \ldots, Y_{\ell}\right)$, by the mean value theorem we obtain

$$
|\mathbb{E} h(X)-\mathbb{E} h(Y)|=\left|\mathbb{E}\left[h^{\prime}(Z)(X-Y)\right]-\mathbb{E}\left[h^{\prime}(0)(X-Y)\right]\right|,
$$


where $Z=Y+U(X-Y)$ for some random variable $U$ in $[0,1]$ and where we have used the fact that the components of $X-Y$ all have expectation 0 . Applying the mean value theorem again as well as the Cauchy-Schwarz inequality yields

$$
\begin{aligned}
|\mathbb{E} h(X)-\mathbb{E} h(Y)| & =\left|\mathbb{E} \sum_{i=1}^{\ell}\left(\frac{\partial h(Z)}{\partial u_{i}}-\frac{\partial h(0)}{\partial u_{i}}\right)\left(X_{i}-Y_{i}\right)\right| \\
& =\left|\mathbb{E} \sum_{i=1}^{\ell} \sum_{j=1}^{\ell} \frac{\partial^{2} h\left(\tilde{Z}^{(i)}\right)}{\partial u_{j} \partial u_{i}} Z_{j}\left(X_{i}-Y_{i}\right)\right| \\
& \leq \sqrt{\mathbb{E} \sum_{i=1}^{\ell}\left(\sum_{j=1}^{\ell} \frac{\partial^{2} h\left(\tilde{Z}^{(i)}\right)}{\partial u_{j} \partial u_{i}} Z_{j}\right)^{2}} \sqrt{\mathbb{E}\|X-Y\|^{2}},
\end{aligned}
$$

with random vectors $\tilde{Z}^{(i)}=U_{i} Z$ and random variables $U_{i} \in[0,1], i=1, \ldots, \ell$. By the fact that $h \in \mathcal{H}$ and the Cauchy-Schwarz inequality, it follows that

$$
\mathbb{E} \sum_{i=1}^{\ell}\left(\sum_{j=1}^{\ell} \frac{\partial^{2} h}{\partial u_{j} \partial u_{i}}\left(\tilde{Z}^{(i)}\right) Z_{j}\right)^{2} \leq \ell^{2} \mathbb{E}\|Z\|^{2} \leq \ell^{2}\left(\mathbb{E}\|X\|^{2}+\mathbb{E}\|Y\|^{2}\right),
$$

which completes the argument.

Proof of Theorem 3. For $i \in\{1, \ldots, \ell\}$, define

$$
\bar{F}_{t}^{(i)}:=t^{-1 / 2} I_{1, t}\left(f_{1}^{(i)}\right), \quad t>0,
$$

and note that $\operatorname{cov}\left[\bar{F}_{t}^{(i)}, \bar{F}_{t}^{(j)}\right]=C_{i j}$. Therefore, from [13, Corollary 4.3] we obtain

$$
d_{3}\left(\left(\bar{F}_{t}^{(1)}, \ldots, \bar{F}_{t}^{(\ell)}\right), N\right) \leq \frac{\ell^{2}}{4} \sum_{i=1}^{\ell} t^{-3 / 2} \int\left|f_{1}^{(i)}(x)\right|^{3} \lambda_{t}(\mathrm{~d} x)=c_{2} t^{-1 / 2}
$$

for some $c_{2}>0$. Lemma 1 implies that

$$
d_{3}\left(\left(\hat{F}_{t}^{(1)}, \ldots, \hat{F}_{t}^{(\ell)}\right),\left(\bar{F}_{t}^{(1)}, \ldots, \bar{F}_{t}^{(\ell)}\right)\right) \leq A_{t}^{1 / 2} B_{t}^{1 / 2},
$$

where

$$
A_{t}:=\ell \sum_{i=1}^{\ell} \mathbb{E}\left(\hat{F}_{t}^{(i)}\right)^{2}+\ell \sum_{i=1}^{\ell} \mathbb{E}\left(\bar{F}_{t}^{(i)}\right)^{2}, \quad B_{t}:=\sum_{i=1}^{\ell} \mathbb{E}\left(\hat{F}_{t}^{(i)}-\bar{F}_{t}^{(i)}\right)^{2} .
$$

The first factor, $A_{t}$, is bounded in $t$. For the second factor we use (26) to obtain

$$
B_{t}=\sum_{i=1}^{\ell} \mathbb{E}\left(\sum_{n=2}^{m_{i}} t^{-n+1 / 2} I_{n, t}\left(f_{n}^{(i)}\right)\right)^{2}=\sum_{i=1}^{\ell} \sum_{n=2}^{m_{i}} t^{-2 n+1} t^{n}\left\|f_{n}^{(i)}\right\|_{n}^{2},
$$

so that $A_{t}^{1 / 2} B_{t}^{1 / 2} \leq c_{3} t^{-1 / 2}, t \geq 1$, for some $c_{3}>0$. Using this estimate in (28) and combining with (27) and the triangle inequality for $d_{3}$, we obtain the result.

Remark 6. The proofs of Theorem 3 and the univariate bound discussed in Remark 4 depend on general Berry-Esseen-type inequalities for Poisson functionals from [13] and [14], that are proven in a slightly more restrictive setting, namely that $(\mathbb{X}, \mathcal{X})$ is a Borel space and $\mu$ is nonatomic. But they are still valid without these assumptions since the proofs only make use of properties of the Malliavin operators that also hold in our more general setting as shown in [10]. 


\section{Poisson flat processes}

In this section we assume that $\eta_{t}$ is a stationary Poisson process of $k$-flats ( $k$-dimensional affine subspaces) in $\mathbb{R}^{d}$, where $d \geq 1$ and $k \in\{0, \ldots, d-1\}$. This is a Poisson process on the space $A(d, k)$ of all $k$-flats, whose distribution is invariant under translation of the flats. Its distribution is determined by the intensity $t>0$ and the directional distribution $\mathbb{Q}$, a probability measure on the space $G(d, k)$ of all $k$-dimensional linear subspaces of $\mathbb{R}^{d}$. In fact, the intensity measure $\lambda_{t}$ of $\eta_{t}$ is equal to

$$
\lambda_{t}(\cdot)=t \int_{G(d, k)} \int_{E^{\perp}} \mathbf{1}_{\{E+x \in \cdot\}} \mathscr{H}^{d-k}(\mathrm{~d} x) \mathbb{Q}(\mathrm{d} E),
$$

where $E^{\perp}$ stands for the orthogonal complement of $E, \mathscr{H}^{d-k}$ denotes the $(d-k)$-dimensional Hausdorff measure, and $\mathbb{Q}$ is a probability measure on $G(d, k)$. We let $\lambda:=\lambda_{1}$. If $\mathbb{Q}$ is the uniform distribution (Haar measure), then $\eta_{t}$ is isotropic, that is, distributionally invariant under rotations. For further details on Poisson flat processes, we refer the reader to [17].

The intersection process of order $m \in \mathbb{N}$ is given as the set of all intersections $E_{1} \cap \cdots \cap E_{m}$ of $m$ pairwise different flats in $\eta_{t}$. To introduce our geometric functionals of the latter process, we let $\mathcal{C}^{d}$ denote the system of all compact subsets of $\mathbb{R}^{d}$, equipped with the Borel $\sigma$-field induced by the Fell topology; see, e.g. [17, Chapter 12]. We consider a measurable family $\mathcal{C}_{0}^{d} \subset \mathcal{C}^{d}$ of sets containing the empty set $\varnothing$ and with the property that $r B \cap E \in \mathcal{C}_{0}^{d}$ for all $B \in \mathcal{C}_{0}^{d}$, all $r>0$, and all affine subspaces $E \subset \mathbb{R}^{d}$. We assume that $\psi: \mathcal{C}_{0}^{d} \rightarrow \mathbb{R}$ is a measurable function with $\psi(\varnothing)=0$ satisfying

$$
\int\left|\psi\left(B \cap E_{1} \cap \cdots \cap E_{m}\right)\right|^{3} \lambda^{m}\left(\mathrm{~d}\left(E_{1}, \ldots, E_{m}\right)\right) \leq C_{B},
$$

for all $B \in \mathcal{C}_{0}^{d}$, where $C_{B} \geq 0$ is a constant only depending on $B$. (By [17, Theorem 12.2.6] the mapping $\left(E_{1}, \ldots, E_{m}\right) \mapsto B \cap E_{1} \cap \cdots \cap E_{m}$ is measurable.) We note here that (29) implies that $\lambda$ is locally finite in the sense that $\lambda(\{E \in A(d, k): B \cap E \neq \varnothing\})<\infty$ for all $B \in \mathcal{C}^{d}$. Since $\psi(\varnothing)=0$, assumption (30) implies the integrability of $\left|\psi\left(B \cap E_{1} \cap \cdots \cap E_{m}\right)\right|^{p}$ with respect to $\lambda^{m}$ for any $p \in(0,3]$. This is enough to settle all integrability issues in this section. Clearly, (30) is implied by the stronger condition

$$
\left|\psi\left(B \cap E_{1} \cap \cdots \cap E_{m}\right)\right| \leq c_{B}, \quad \lambda^{m} \text {-a.e. }\left(E_{1}, \ldots, E_{m}\right), \quad B \in \mathcal{C}_{0}^{d},
$$

for some $c_{B} \geq 0$ depending on $B$. In particular, (31) is satisfied in our examples below.

Define a random field $\zeta_{t}:=\left\{\zeta_{t}(B): B \in \mathcal{C}_{0}^{d}\right\}$ by

$$
\zeta_{t}(B):=\frac{1}{m !} \int \psi\left(B \cap E_{1} \cap \cdots \cap E_{m}\right) \eta_{t}^{(m)}\left(\mathrm{d}\left(E_{1}, \ldots, E_{m}\right)\right), \quad B \in \mathcal{C}_{0}^{d} .
$$

Since $\eta_{t}$ has only atoms of size one (by (29)) we can identify $\eta_{t}$ with its support, and integration with respect to $\eta_{t}^{(m)}$ corresponds to summation over all $m$-tuples $\left(E_{1}, \ldots, E_{m}\right) \in \eta_{t}^{m}$ with pairwise different entries. For $A, B \in \mathcal{C}_{0}^{d}$, define

$C(A, B)$

$$
\begin{aligned}
:=\frac{1}{((m-1) !)^{2}} \int & \left(\int \psi\left(A \cap E_{1} \cap E_{2} \cap \cdots \cap E_{m}\right) \lambda^{m-1}\left(\mathrm{~d}\left(E_{2}, \ldots, E_{m}\right)\right)\right) \\
& \times\left(\int \psi\left(B \cap E_{1} \cap E_{2}^{\prime} \cap \ldots \cap E_{m}^{\prime}\right) \lambda^{m-1}\left(\mathrm{~d}\left(E_{2}^{\prime}, \ldots, E_{m}^{\prime}\right)\right)\right) \lambda\left(\mathrm{d} E_{1}\right) .
\end{aligned}
$$


If $m=1$, this has to be read as

$$
C(A, B)=\int \psi\left(A \cap E_{1}\right) \psi\left(B \cap E_{1}\right) \lambda\left(\mathrm{d} E_{1}\right) .
$$

It can be checked directly that $C(\cdot, \cdot)$ is positive semidefinite. Therefore, we can consider a centred Gaussian field $\xi:=\left\{\xi(B): B \in \mathcal{C}_{0}^{d}\right\}$ with this covariance function.

Define $\hat{\zeta}_{t}(B):=t^{-(m-1 / 2)}\left(\zeta_{t}(B)-\mathbb{E} \zeta_{t}(B)\right), t>0, B \in \mathcal{C}_{0}^{d}$.

Theorem 4. Let $\ell \geq 1$ and $B_{1}, \ldots, B_{\ell} \in \mathcal{C}_{0}^{d}$. Then

$$
d_{3}\left(\left(\hat{\zeta}_{t}\left(B_{1}\right), \ldots, \hat{\zeta}_{t}\left(B_{\ell}\right)\right),\left(\xi\left(B_{1}\right), \ldots, \xi\left(B_{\ell}\right)\right)\right) \leq c\left(B_{1}, \ldots, B_{\ell}\right) t^{-1 / 2}, \quad t \geq 1,
$$

for some constant $c\left(B_{1}, \ldots, B_{\ell}\right)$. In particular,

$$
\left\{\hat{\zeta}_{t}(B): B \in \mathcal{C}_{0}^{d}\right\} \stackrel{\mathrm{D}}{\rightarrow}\left\{\xi(B): B \in \mathcal{C}_{0}^{d}\right\} \quad \text { as } t \rightarrow \infty,
$$

in the sense of convergence of finite-dimensional distributions.

Proof. This is a direct consequence of Theorem 3.

Alternatively, we can approach the central limit problem in another closely related setting. Instead of increasing the intensity parameter $t$, we can also fix $t$ (for simplicity we take $t=1$ ) and increase the size $r$ of the observation window. If we assume additionally that the considered function $\psi$ is homogeneous of degree $\alpha \in \mathbb{R}$, that is,

$$
\psi(r B)=r^{\alpha} \psi(B), \quad B \in \mathcal{C}_{0}^{d}, \quad r>0,
$$

both approaches are equivalent. Define a random field $\tilde{\zeta}_{r}:=\left\{\tilde{\zeta}_{r}(B): B \in \mathcal{C}_{0}^{d}\right\}$ with $\tilde{\zeta}_{r}(B)=$ $r^{-(m-1 / 2)(d-k)-\alpha}\left(\zeta_{1}(r B)-\mathbb{E} \zeta_{1}(r B)\right)$.

Corollary 4. Assume that (32) holds; let $\ell \geq 1$ and $B_{1}, \ldots, B_{\ell} \in \mathcal{C}_{0}^{d}$. Then

$$
d_{3}\left(\left(\tilde{\zeta}_{r}\left(B_{1}\right), \ldots, \tilde{\zeta}_{r}\left(B_{\ell}\right)\right),\left(\xi\left(B_{1}\right), \ldots, \xi\left(B_{\ell}\right)\right)\right) \leq c\left(B_{1}, \ldots, B_{\ell}\right) r^{-(d-k) / 2}, \quad r \geq 1,
$$

for some constant $c\left(B_{1}, \ldots, B_{\ell}\right)$. In particular, $\left\{\tilde{\zeta}_{r}(B): B \in \mathcal{C}_{0}^{d}\right\} \stackrel{\mathrm{D}}{\rightarrow}\left\{\xi(B): B \in \mathcal{C}_{0}^{d}\right\}$ as $r \rightarrow \infty$ in the sense of finite-dimensional distributions.

Proof. The special structure (29) of the intensity measure $\lambda$ implies the well-known scaling property

$$
\mathbb{P}\left(\eta_{t} \in \cdot\right)=\mathbb{P}\left(t^{-1 /(d-k)} \eta_{1} \in \cdot\right), \quad t>0,
$$

where $a \eta_{1}:=\left\{a E: E \in \eta_{1}\right\}$ for $a>0$. Since $\psi$ is homogeneous, we obtain, for all $B \in \mathcal{C}_{0}^{d}$ and $r>0$,

$$
\begin{aligned}
\zeta_{1}(r B) & =\frac{1}{m !} r^{\alpha} \int \psi\left(B \cap r^{-1} E_{1} \cap \cdots \cap r^{-1} E_{m}\right) \eta_{1}^{(m)}\left(\mathrm{d}\left(E_{1}, \ldots, E_{m}\right)\right) \\
& =\frac{1}{m !} r^{\alpha} \int \psi\left(B \cap E_{1} \cap \cdots \cap E_{m}\right) \eta_{r^{1 /(d-k)}}^{(m)}\left(\mathrm{d}\left(E_{1}, \ldots, E_{m}\right)\right),
\end{aligned}
$$

where the second identity holds in distribution jointly in $B$. Hence, we can apply Theorem 3 with $g_{1}(t)=\cdots=g_{\ell}(t):=(m !)^{-1} t^{\alpha(d-k)}$ and then replace $t$ by $r^{1 /(d-k)}$.

Remark 7. It follows from (21) (with $g_{i}(t)=g_{j}(t)=1 / m !$ ) that

$$
\operatorname{cov}\left[\zeta_{t}(A), \zeta_{t}(B)\right]=\sum_{n=1}^{m} \frac{1}{n !((m-n) !)^{2}} V_{t}(A, B, n), \quad A, B \in \mathcal{C}_{0}^{d},
$$


where

$$
\begin{aligned}
& V_{t}(A, B, n) \\
& :=t^{2 m-n} \int\left[\int \psi\left(A \cap E_{1} \cap \cdots \cap E_{n} \cap E_{n+1} \cap \cdots \cap E_{m}\right) \lambda^{m-n}\left(\mathrm{~d}\left(E_{n+1}, \ldots, E_{m}\right)\right)\right. \\
& \left.\quad \times \int \psi\left(B \cap E_{1} \cap \cdots \cap E_{n} \cap E_{n+1} \cap \cdots \cap E_{m}\right) \lambda^{m-n}\left(\mathrm{~d}\left(E_{n+1}, \ldots, E_{m}\right)\right)\right] \\
& \quad \times \lambda^{n}\left(\mathrm{~d}\left(E_{1}, \ldots, E_{n}\right)\right) .
\end{aligned}
$$

In accordance with Theorem 4, we therefore obtain

$$
\lim _{t \rightarrow \infty} t^{-(2 m-1)} \operatorname{cov}\left[\zeta_{t}(A), \zeta_{t}(B)\right]=C(A, B)
$$

We now present a few examples to which Theorem 4 as well as Corollary 4 can be applied.

Example 3. Assume that $m(d-k) \leq d$. Assume further that $\mathcal{C}_{0}^{d}=\mathcal{C}^{d}$ and that $\psi$ is the $(d-m(d-k))$-dimensional Hausdorff measure on $\mathbb{R}^{d}$ restricted to $\mathcal{C}^{d}$. Then (32) holds with $\alpha=d-m(d-k)$. Assumption (31) holds because for $\lambda^{m}$-a.e. $\left(E_{1}, \ldots, E_{m}\right) \in A(d, k)^{m}$ the intersection $E_{1} \cap \cdots \cap E_{m}$ is either empty or has dimension $d-m(d-k)$. This follows (recursively) from the argument given in [17, p. 130].

Example 4. Assume that $\mathcal{C}_{0}^{d}=\mathcal{C}^{d}$ and that $\psi(B)=\mathbf{1}_{\{B \neq \varnothing\}}$. Then (32) holds with $\alpha=0$ while (31) holds with $c_{B}=1$.

Examples 3 and 4 have been studied in [4] and [5] for the case $k=d-1$. Our results add to [4, Theorems 3.1 and 4.1] in several ways. While the latter results are multivariate central limit theorems for the $d$ possible values of the number $m$ of intersections but a fixed (convex) test set $B$, we fix $m$ but study $\zeta_{t}(B)$ (respectively $\zeta_{1}(r B)$ ) as a function of $B$. Furthermore, we consider processes of flats and not only hyperplanes. Moreover, we obtain Berry-Esseen-type bounds on the distance $d_{3}$ and can allow for a considerably larger class of functionals $\psi$. It is also possible to apply Theorem 3 to the vector-valued processes arising by varying $m$. This would constitute a complete generalization of [4]. In order to avoid heavy notation we have refrained from doing so.

We continue with further examples of functionals $\psi$ satisfying (31) and (32). The convex ring $\mathcal{R}^{d}$ is the system of all (possible empty) unions of convex and compact subsets of $\mathbb{R}^{d}$.

Example 5. Assume that $\mathcal{C}_{0}^{d}=\mathcal{R}^{d}$ and that $\psi$ is the intrinsic volume $V_{\alpha}$, where $\alpha \in\{0, \ldots, d\}$; see, e.g. [17]. Then (32) holds. Assumption (31) follows from the fact that $V_{\alpha}(B \cap E) \leq V_{\alpha}(B)$ for any convex and compact $B \subset \mathbb{R}^{d}$ and any affine subspace $E \subset \mathbb{R}^{d}$. By additivity of $V_{\alpha}$ (see, e.g. [17, Section 14.2]) inequality (31) can be established for the whole convex ring.

In contrast to the previous examples, the next functionals are not additive.

Example 6. Assume that $\mathcal{C}_{0}^{d}=\mathcal{R}^{d}$ and $\alpha \in\{0, \ldots, d-1\}$. Let $\Theta_{\alpha}(A, \cdot)$ be the support measure of $A \in \mathcal{R}^{d}$; see [17, Section 14.2]. This is a signed measure on the product of $\mathbb{R}^{d}$ and the unit sphere $\mathbb{S}^{d-1}$ such that $\Theta_{\alpha}\left(A, \mathbb{R}^{d} \times \mathbb{S}^{d-1}\right)=V_{\alpha}(A)$. Fix a measurable set $U \subset \mathbb{S}^{d-1}$ and assume that

$$
\psi(A)=\int \mathbf{1}_{\{(x, u) \in N(A), u \in U\}} \Theta_{\alpha}(A, \mathrm{~d}(x, u)),
$$

where $N(A)$ is the unit normal bundle of $A$. This consists of all pairs $(x, u) \in \mathbb{R}^{d} \times \mathbb{S}^{d-1}$ that occur as a unique nearest point and the associated direction of a point in the complement of $A$; 
see [6]. The homogeneity (32) follows from the homogeneity of the (nonnegative) measure $\mathbf{1}_{\{(x, u) \in N(A)\}} \Theta_{\alpha}(A, d(x, u))$; see [6, Proposition 4.9]. Assumption (31) follows similarly as in Example 5 from the additivity of $\Theta_{\alpha}(A, \cdot)$ in $A \in \mathcal{R}^{d}$.

Example 7. Consider the case where $\mathcal{C}_{0}^{d}$ is the space of compact convex subsets of $\mathbb{R}^{d}$, fix $\alpha \in\{0, \ldots, d\}$ and $\beta \geq 0$, and let $\psi$ be $V_{\alpha}^{\beta}$, the power $\beta$ of the intrinsic volume of order $\alpha$. In the case $\alpha=1$ and $\beta=n \in \mathbb{N}, \psi$ corresponds to the $n$th chord-power integral, which is frequently studied in integral geometry; see [17, Chapter 8.6]. Clearly, (32) is satisfied with $\alpha \beta$ there and assumption (31) follows as in Example 5 from the fact that $V_{\alpha}^{\beta}(B \cap E) \leq V_{\alpha}^{\beta}(B)$ for any convex and compact $B \subset \mathbb{R}^{d}$ and any affine subspace $E \subset \mathbb{R}^{d}$.

Remark 8. If $\psi \geq 0$, then $\operatorname{cov}\left[\zeta_{t}(A), \zeta_{t}(B)\right] \geq 0$ and $C(A, B) \geq 0$ for all $A, B \in \mathcal{C}_{0}^{d}$. This is the case in Examples 3, 4, and 7. Taking the system of convex sets as $\mathcal{C}_{0}^{d}$, this is also the case in Examples 5 and 6. If additionally $m \geq 2$, Corollary 2 shows that the moment generating functions of the functionals under consideration do not exist.

\section{Acknowledgement}

The authors would like to thank two anonymous referees for a number of valuable comments, which were helpful for us to improve the text.

\section{References}

[1] Bhattacharya, R. N. and Ghosh, J. K. (1992). A class of $U$-statistics and asymptotic normality of the number of $k$-clusters. J. Multivariate Anal. 43, 300-330.

[2] Billingsley, P. (1979). Probability and Measure. John Wiley, New York.

[3] Decreusefond, L., Ferraz, E., Randriambololona, H. and Vergne, A. (2014). Simplicial homology of random configurations. To appear in Adv. Appl. Prob.

[4] Heinrich, L. (2009). Central limit theorems for motion-invariant Poisson hyperplanes in expanding convex bodies. Rend. Circ. Mat. Palermo (2), Suppl. 81, 187-212.

[5] Heinrich, L., Schmidt, H. And Schmidt, V. (2006). Central limit theorems for Poisson hyperplane tessellations. Ann. Appl. Prob. 16, 919-950.

[6] Hug, D., Last, G. AND WeIL, W. (2004). A local Steiner-type formula for general closed sets and applications. Math. Z. 246, 237-272.

[7] Kallenberg, O. (2002). Foundations of Modern Probability, 2nd edn. Springer, New York.

[8] Lachièze-Rey, R. and Peccati, G. (2013). Fine Gaussian fluctuations on the Poisson space, I: contractions, cumulants and geometric random graphs. Electron. J. Prob. 18, 32.

[9] Lachièze-Rey, R. ANd Peccati, G. (2013). Fine Gaussian fluctuations on the Poisson space, II: rescaled kernels, marked processes and geometric $U$-statistics. Stoch. Process. Appl. 123, 4186-4218.

[10] Last, G. and Penrose, M. D. (2011). Poisson process Fock space representation, chaos expansion and covariance inequalities. Prob. Theory Relat. Fields 150, 663-690.

[11] Minh, N. T. (2011). Malliavin-Stein method for multi-dimensional $U$-statistics of Poisson point processes. Preprint. Available at http://uk.arxiv.org/abs/1111.2140.

[12] Peccati, G. and Taqqu, M. S. (2011). Wiener Chaos: Moments, Cumulants and Diagrams. Springer, Milan.

[13] Peccati, G. And Zheng, C. (2010). Multi-dimensional Gaussian fluctuations on the Poisson space. Electron. J. Prob. 15, 1487-1527.

[14] Peccati, G., Solé, J. L., Taqqu, M. S. and Utzet, F. (2010). Stein's method and normal approximation of Poisson functionals. Ann. Prob. 38, 443-478.

[15] Penrose, M. (2003). Random Geometric Graphs. Oxford University Press.

[16] Reitzner, M. And Schulte, M. (2013). Central limit theorems for $U$-statistics of Poisson point processes. Ann. Prob. 41, 3879-3909.

[17] SchneIder, R. AND WeIL, W. (2008). Stochastic and Integral Geometry. Springer, Berlin.

[18] Schulte, M. and Thäle, C. (2014). Distances between Poisson k-flats. Methodol. Comput. Appl. Prob. 16, 311-329.

[19] Surgailis, D. (1984). On multiple Poisson stochastic integrals and associated Markov semigroups. Prob. Math. Statist. 3, 217-239. 